\title{
A GAS ANALYSIS PIPETTE FOR DIFFICULT ABSORPTIONS
}

\author{
By Martin Shepherd
}

\section{ABSTRACT}

This note describes a bubbling pipette which employs a thin platinum distributor plate perforated with 200 holes of $0.06 \mathrm{~mm}$ diameter. This distributor plate breaks the influent gas stream into many small bubbles, and effects intimate contact between a liquid reagent and the gas to be absorbed. The efficiency of the pipette has been compared with the ordinary bubbling type and with the Dennis-Friedrichs pipette. Oxygen is completely removed from air during three passages into alkaline pyrogallol contained in both the new pipette and the Dennis-Friedrichs pipette, whereas 10 passages were required for the ordinary type. The new pipette possesses some advantages over the Dennis-Friedrichs design, mainly lower cost, simplicity of construction, less fragility, and the fact that no special molds are required for its construction.

\section{CONTENTS}

II. Development of the pipette.

III. Eescription of the pipette of the pipette

IV. Comparison of the distributor and Dennis-Friedrichs pipette

\section{DEVELOPMENT OF THE PIPETTE}

A number of gas analysis pipettes have been designed to bring the gas and reagent into intimate contact. Pipettes of the bubbling type possess distinct advantages in this respect. The ordinary bubbling pipette which appears at present in various modifications and under various names was first designed by Hankus in $1899 .{ }^{1}$ The best modifications of this pipette so far described are one adapted from the Friedrichs gas washing bottle by L. M. Dennis ${ }^{2}$ (fig. $1(b)$ ) and one designed by Nowicki ${ }^{3}$ and improved by Heinz. ${ }^{4}$ Dennis reports the complete removal of oxygen from air after three passages into alkaline pyrogallol contained in the Dennis-Friedrichs and NowickiHeinz pipettes.

When the Jena glass sintered filters were developed, the author constructed a pipette in which the gas inlet tube of an ordinary bubbling pipette was bent upward and tipped with a coarse Jena sintered filter. The gas stream was thus finely divided, and fairly rapid absorption resulted; but the filter plate trapped a measurable amount of gas.

Development of the idea was continued by using a platinum plate perforated with numerous small holes. It was found that when the

1 Hankus, E. E., Osterr. Chem. Ztg., 47, p. 81; 1899; J. Gasbel., 49, p. 367; 1906.

2 Dennis, L. M., Gas Analysis, p. 81'(fig. 47), The Macmillan Co., New York; 1913.

3 Nowicki, Österr. Z. Berg. Hüttenw., 53, p. 337; 1905.

4 Heinz, J. Gasbel., 49, p. 367; 1906. 
plate was placed so that the burrs resulting from punching these holes were on the efflux side and the grease film ordinarily present on the platinum surface was removed, the gas stream then issued from the plate in a series of fine bubbles. With ordinary teçhnic,

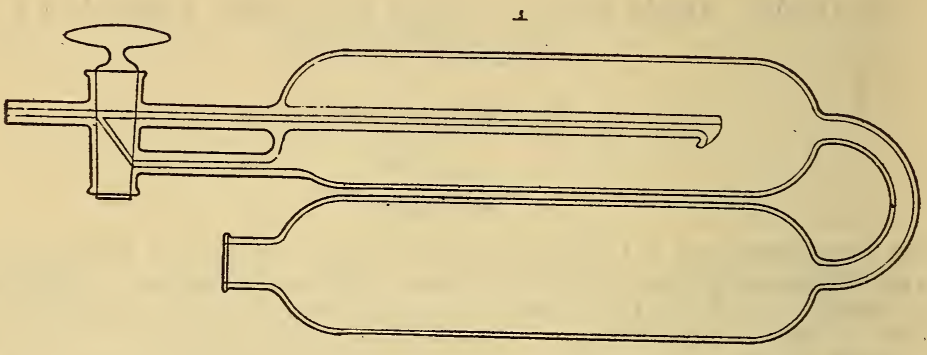

3
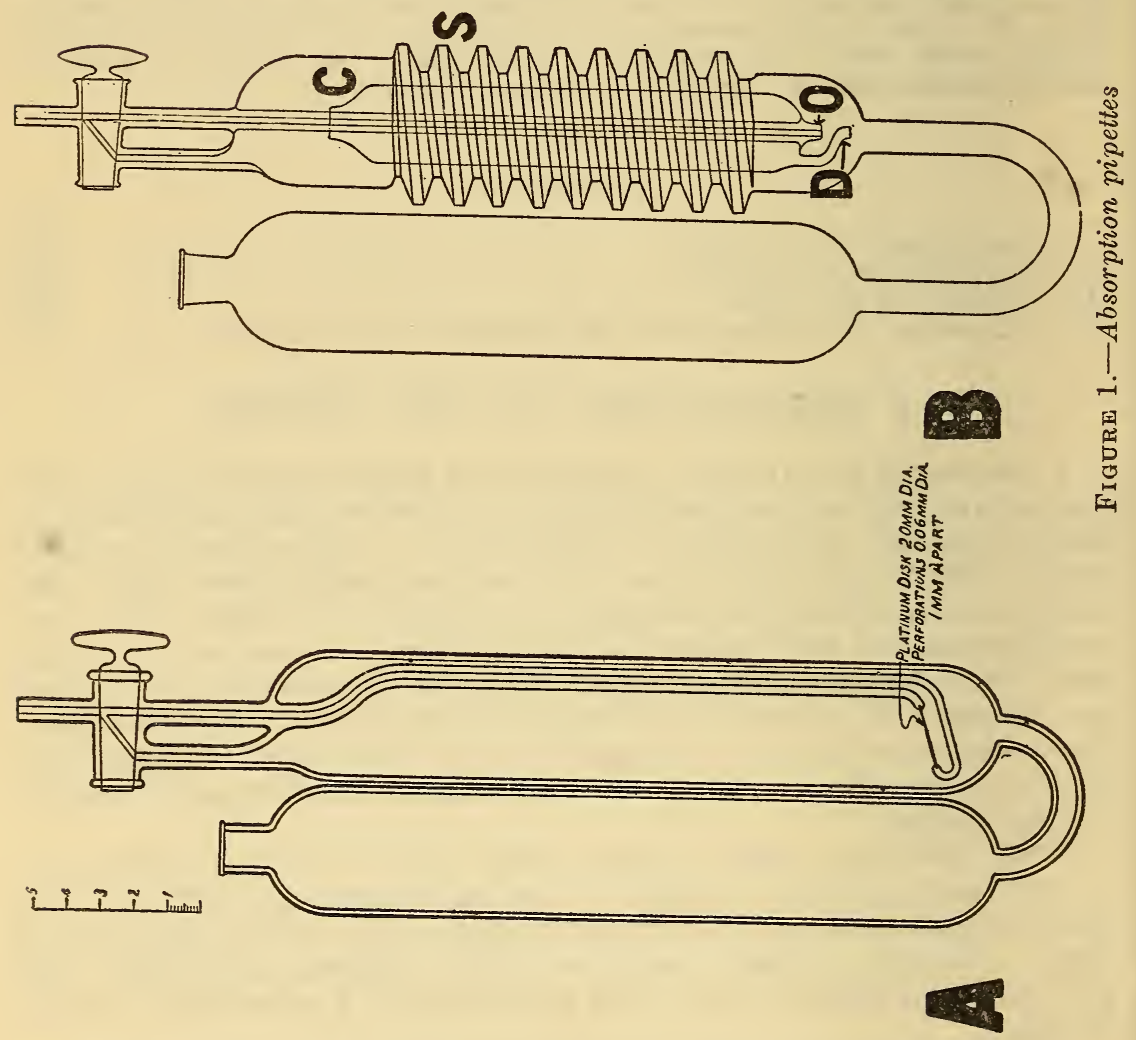

however, it was not possible to obtain holes of sufficiently uniform diameter. Accordingly the perforated plates were obtained from a company engaged in manufacturing spinnerets for the rayon industry. These proved to be entirely satisfactory. 


\section{DESCRIPTION OF THE PIPETTE}

The pipette constructed with this gas distributing tip is shown in Figure $1(a)$. The perforated plate is $0.05 \mathrm{~mm}$ thick and $20 \mathrm{~mm}$ in diameter. The holes are $0.06 \mathrm{~mm}$ in diameter $\pm 0.003 \mathrm{~mm}$, spaced $1 \mathrm{~mm}$ apart, and extend within $2 \mathrm{~mm}$ of the outer edge of the plate. There are 212 evenly spaced holes in such a plate. The burrs resulting from drilling were not removed. The plate is sealed in to the flat dish-shaped end of the gas inlet tube which is placed at an angle in order to prevent trapping the return gas.

About $30 \mathrm{~mm}$ mercury pressure is required to force the gas through the perforated plate. When in use the leveling bulb attached to the burette is placed slightly above the upper stopcock of the burette, and the lower burette stopcock opened to permit free passage of mercury from the leveling bulb. Under these conditions, a $100 \mathrm{ml}$ sample passes into the pipette in about 20 seconds. The gas issues from the perforated plate in very small bubbles which apparently do not coalesce. A froth consisting of many very small bubbles separated by liquid films is formed on the surface of the reagent. This layer of froth is about $2 \mathrm{~cm}$ deep, when an alkaline pyrogallol solution is used, and some absorption must take place here as well as in the actual passage through the reagent. The froth disappears immediately when the gas flow through the perforated plate has ceased.

At the beginning of an absorption, the gas passes through approximately $7 \frac{1}{2}$ inches of reagent. This depth of liquid decreases to about $2 \frac{1}{2}$ inches for a $100 \mathrm{ml}$ sample. In the Dennis-Friedrichs pipette, the effective depth of reagent is approximately 50 inches, decreasing only 2 inches with the passage of a $100 \mathrm{ml}$ sample. In spite of this fact the absorption in the distributing pipette is slightly more rapid.

When a viscous reagent is used, the liquid returns slowly through the small orifices of the distributor plate and, if the ordinary technic is used, some time is lost in displacing the gas in the inlet capillary when it is desired to return the sample to the burette for final measurement. This difficulty is overcome by allowing the stopcock of the pipette to remain adjusted in the position for delivery of gas into the distributing plate and reducing the pressure in the burette about $2 \mathrm{~cm}$. The reagent then flows through the perforated plate and into the capillary within a few seconds, but rises in the capillary at a very easily controlled rate. The pipette stopcock is then turned to the normal position for displacement of the gas sample, which may be returned to the burette within the same time that is required for the plain bubbling type of pipette.

\section{EFFICIENCY OF THE PIPETTE}

The efficiency of the pipette was determined by the absorption of oxygen from air, and of oxygen from a mixture of oxygen and nitrogen containing about 95 per cent of oxygen. The reagent used was an alkaline pyrogallol solution recommended by Anderson. ${ }^{5}$ The absorption of oxygen by alkaline pyrogallol is somewhat slower than by other reagents which have been recommended, but for this reason it is suitable for a study of relative efficiencies. Dennis ${ }^{6}$ also used

Anderson, R. P., Ind. Eng. Chem., 7, p. 588; 1915.

Dennis, L. M., Gas Analysis, p. 82. 'The Macmillan Co., New York; 1913. 
alkaline pyrogallol in comparing the efficiencies of several pipettes, and his results will be given for the sake of comparison.

The assembly diagram of the gas analysis apparatus used is shown in Figure 2. The burette $B$, manometer $M$, and pipette $P$ are con-

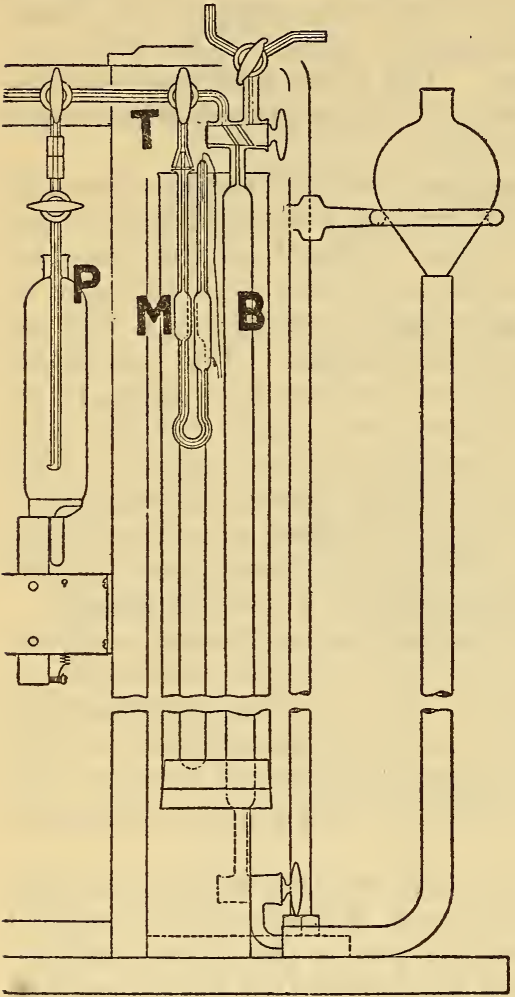

FIGURE 2.-Assembly of apparatus nected to the train $T$ so that pressure may be balanced simultaneously in burette and train, and gas contained over the arm of the manometer connected to the train may be completely displaced into the train and burette before each passage of a sample to the pipette. A very small dead capillary space results.

It is not possible to pass a $100 \mathrm{ml}$ sample into the DennisFriedrichs pipette within the $20 \mathrm{sec}-$ onds required for complete passage into the distributor pipette. If the passage is made in less than $45 \mathrm{sec}-$ onds, the gas collecting at the bottom of the Friedrichs spiral may be forced into the reservoir arm of the pipette and so lost. Dennis compared his pipette with others employing 1-minute passages into each pipette. The present tests were made at various rates, but some of the data are directly comparable in this respect. The data for the removal of oxygen from air are given in Table 1, and those for the removal of oxygen from 95 per cent $\mathrm{O}_{2}$ in $\mathrm{N}_{2}$ are given in Table 2.

TABLE 1.-Percentage of the total oxygen content of air which is removed during each passage into various types of bubbling pipettes containing alkaline pyrogallol

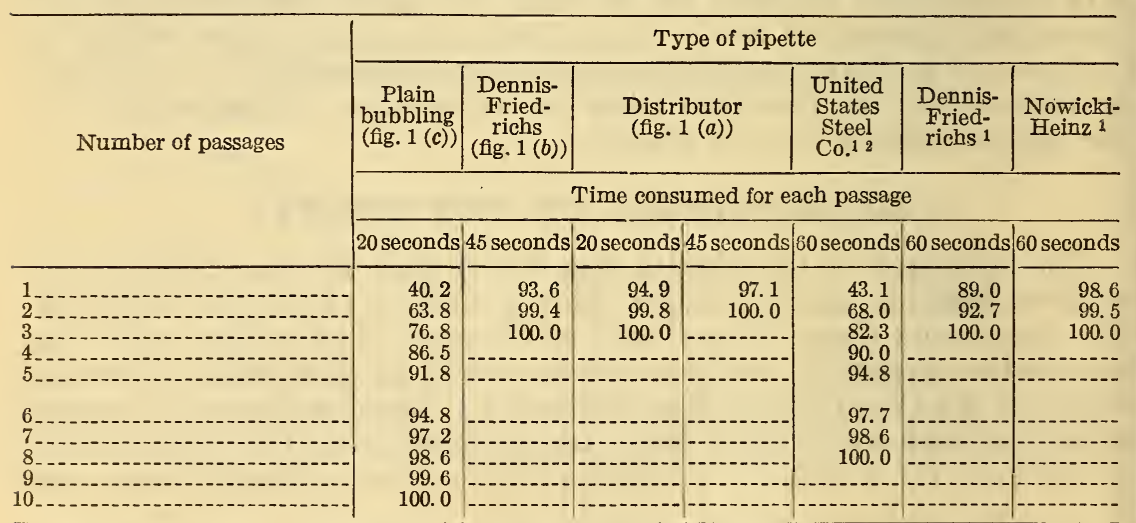

1 Results obtained by L. M. Dennis, Gas Analysis, p. 82. The Macmillan Co., New York; 1913.

2 A plain bubbling pipette of slightly greater length than the one shown in fig. 1 (c). 
TABLE 2.-Percentage of the total oxygen content of a 95 per cent $\mathrm{O}_{2}+5$ per cent $\mathrm{N}_{2}$ mixture which is removed during each passage into the distributor and DennisFriedrich's pipettes

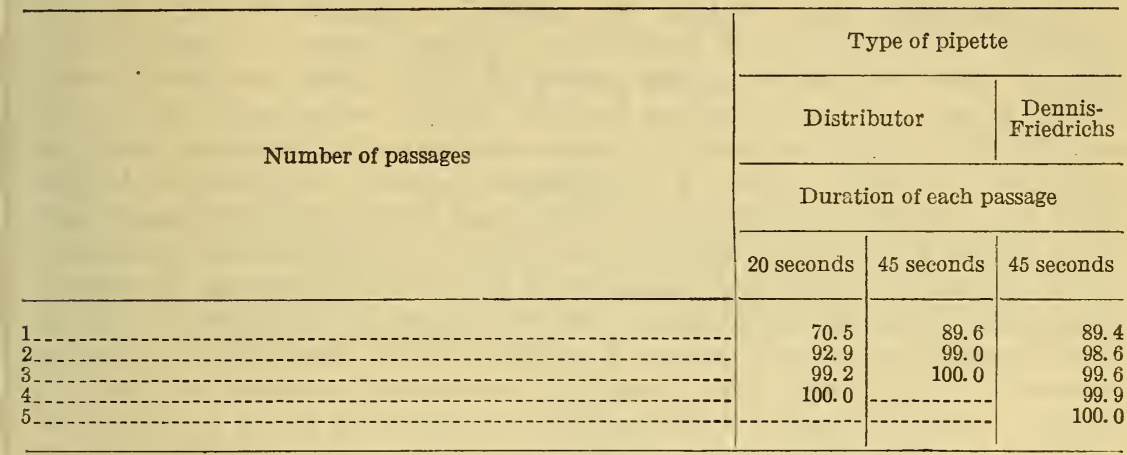

The tabulated data show the following significant facts:

1. Oxygen is removed from air more quickly in the distributor pipette than in the Dennis-Friedrichs pipette. This is true for both the 20 and 45 second rates of passage. Three passages are required to complete the removal of oxygen at the 20 -second rate, and two passages at the 45-second rate. The Dennis-Friedrichs pipette requires three passages at the 45 -second rate. Ten passages are required in the plain bubbling type pipette (fig. $1(c)$ ) at the 20-second rate.

2. In the case of the 95 per cent oxygen sample, the distributor pipette required four passages at the 20 -second rate and three passages at the 45-second rate. The Dennis-Friedrichs pipette required five passages at the slower rate. A small volume of gas remained after the second passage (about $5 \mathrm{ml}$ ) and the efficiency of the Dennis pipette was considerably lowered thereafter.

\section{COMPARISON OF THE DISTRIBUTOR AND DENNIS- FRIEDRICHS PIPETTES}

While the distributor pipette has been shown to be more rapid, a comparison of the two pipettes should include other considerations. These may be briefly stated.

1. The outer spiral shell ( $S$ of fig. $1(b)$ ) of the Dennis-Friedrichs pipette is blown in a mold. The inner surface is then ground to fit the inside cylinder $C$, which terminates in a liquid inlet, $D$, and a gas outlet, $O$. The capillary tube through which the gas passes in to the pipette requires two ring seals, one at the dome of the pipette and one at the bottom of the inner cylinder. This construction results in the following disadvantages:

(a) The construction is more complicated and expensive than that of the distributor pipette.

(b) The mold blown spiral is apt to be fragile. A number have been found broken in shipment. The outer shells were of uneven thickness, and some were extremely thin in places.

(c) In constructing the pipette, the stopcock and the capillary gas inlet must be sealed to the dome, which is drawn out from the mold $106307^{\circ}-30-2$ 
blown shell. Proper annealing is then evidently difficult, since a number of pipettes have cracked at this point during normal use.

$(d)$ The liquid inlet $D$ of the cylinder $C$ is ordinarily made with an inside diameter that varies by several millimeters. The inside diameter of this tube is somewhat critical, since it should offer slightly more resistance to the return of liquid than the spiral itself. If this is not the case, the gas bubbles which form in the flat spiral are difficult to displace when a viscous reagent-for example, alkaline pyrogallol-is used. One of the Dennis pipettes trapped gas which could not be displaced from the spiral, and others required watching.

(e) When gas is first introduced into the pipette it accumulates at the bottom of the spiral until some has passed through the spiral itself and displaced the reagent from the dome of the pipette. Care must be used to prevent loss of a portion of the sample into the side reservoir. This limits the speed of passage of a sample into the pipette. It has already been pointed out that 45 to 60 seconds are required for a $100 \mathrm{ml}$ sample, while the same volume can be passed into the distributor pipette in 20 seconds. Furthermore, when a small residual volume is passed into the Dennis pipette, care must be taken to avoid trapping bubbles of the gas in the capillary gas outlet 0 , which will result in the formation of slugs of the reagents in this capillary when the gas is displaced into the burette.

$(f)$ Molds for the construction of the Dennis pipette are not available in this country, and the pipette must be imported at present.

2. The fine orifices of the distributor plate of the new pipette may possibly become clogged by precipitation from some reagents. This difficulty has not been experienced with Anderson's solution.

Washington, January 4, 1930. 